Steven C. Hall MD

Department of Anesthesiology

Children's Memorial Plaza

Chicago, Illinois 60614

USA

REFERENCE

1 Roth $A G$, Wheeler M, Stevenson GW, Hall SC.

Comparison of a rigid laryngoscope with the ultrathin fiberoptic laryngoscope for tracheal intubation in infants.

Can J Anaesth 1994; 41: 1069-73.

\section{Attenuation of the pressor response to laryngoscopy - misquotation}

To the Editor:

I read the article by Wig et al. ${ }^{1}$ which showed an attenuating effect of calcium entry blockers upon the pressor response associated with laryngoscope and tracheal intubation. However, they erroneously quoted our article ${ }^{2}$ and stated that we could not observe any beneficial effect of verapamil. Indeed, we did find it. There is also an additional error in the statement concerning the peak effect of verapamil, which is apparent nearly one minute after $i$ injection. ${ }^{3-5}$

\section{Toshiaki Nishikawa MD}

Department of Anesthesiology

Institute of Clinical Medicine

University of Tsukuba

Tsukuba City, Ibaraki 305

Japan

\section{REFERENCES}

1 Wig J, Sharma M, Baichoo N. Agarwal A. Nicardipine and verapamil attenuate the pressor response to laryngoscopy and intubation. Can J Anaesth 1994; 41: 1185-8.

2 Nishikawa T, Namiki A. Attenuation of the pressor response to laryngoscopy and tracheal intubation with intravenous verapamil. Acta Anaesthesiol Scand 1989; 33: 232-5.

3 Zimpfer M, Fitzal S, Tonczar L. Verapamil as a hypotensive agent during neurolept anaesthesia. Br J Anaesth 1981; 53: 885-9.

4 Nishikawa $T$, Namiki $A$. The effects of verapamil on cerebrospinal fluid pressure in surgical patients. Masui 1987; 1 : 132-6.

5 Singh $B N$, Roche $A H G$. Effects of intravenous verapamil on hemodynamics in patients with heart disease. Am Heart J 1977; 94: 593-9.

\section{$R E P L Y$}

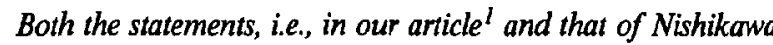
et al. (Ref No. 2) regarding attenuation of pressor responses are partly correct. In Nishikawa's article, tachycardia could not be attenuated. However, an increase in MAP could only be attenuated for the first 5-10 min. Later, the difference in the rise in MAP in both the control and verapamil-treated groups was insignificant. We did not fully elucidate this fact. Also, we apologize for the second error and will be more careful in future.

Jyotsna Wig MD

Chandigarh

India

\section{Laryngoscopy and arrhythmias in children}

To the Editor:

We would like to report our observations involving the use of halothane during induction, laryngoscopy, and tracheal intubation in children with supraventricular tachycardia (SVT) syndromes. With the establishment of a paediatric cardiac electrophysiological study (EPS) diagnostic laboratory at our institution, that included radiofrequency ablation (RFA) procedures when appropriate, the anaesthesia department was asked to provide general anaesthesia for these children. This was due to the anticipated prolonged nature of the cases (4-12 hr), the potential for complications (e.g., SVT requiring cardioversion) and the desire to have a motionless, stable child during placement of the multiple catheters.

Since it was necessary to enable the cardiologist to elicit SVT for both diagnostic and therapeutic reasons, we initially selected halothane as our primary anaesthetic due to its reputation for arrhythmogenicity as well as our familiarity with the agent in routine paediatric anaesthetic practice. Dealing with a patient group that sought RFA due to life-threatening arrhythmias, medication failures or complications, or desire for a definitive correction, we were concerned about eliciting SVT during induction and intubation. We selected a protocol consisting of benzodiazepine premedication (diazepam 0.2 $\mathrm{mg} \cdot \mathrm{kg}^{-1}$ ), placement of an iv catheter upon arrival in the catheterization laboratory, and $i v$ induction with thiopentone $4 \mathrm{mg} \cdot \mathrm{kg}^{-1}$, vecuronium $0.1 \mathrm{mg} \cdot \mathrm{kg}^{-1}$, and then inhalational halothane, $1.5 \%$ end-tidal concentration, $\mathrm{N}_{2} \mathrm{O}$ $3 \mathrm{~L} \cdot \mathrm{min}^{-1}$ and $\mathrm{O}_{2} 2 \mathrm{~L} \cdot \mathrm{min}^{-1}$ with $\mathrm{PETCO}_{2} 35-40$ $\mathrm{mmHg}$. Continuous monitoring for SVT was recorded, with hardcopy printouts prior to induction, after induction with achievenment of end-tidal halothane concentrations of $1.5 \%$ for three minutes, and again immediately after laryngoscopy and intubation.

No arrhythmias were observed during induction, lar- 